\title{
A METHODOLOGY FOR DOCUMENTING HAND POSITIONS AND FORCES DURING MANUAL WORK*
}

\author{
T. J. Armstrong, D. B. Chaffin and J. A. Follke
}

Department of Industrial and Operations Engineering, 2254 G. G. Brown Laboratory,

The University of Michigan, Ann Arbor, MI 48109. U.S.A

In the course of a study to determine work methods associated with occupational wrist injuries, a low cost method was developed for estimating the frequencies that various hand positions and the corresponding hand forces were used for certain manual jobs. Hand positions were documented by filming the hands of a worker with a super $8 \mathrm{~mm}$ motion picture camera at four frames per sec. (See Fig. 1). The film was then reviewed frame by frame through several work cyclest. The positions from each frame were noted, plotted and summarized as is shown in Fig. 2.

The hand forces were estimated from the integrated

* Received 9 June 1978.

+ Lafayette Super $8 \mathrm{~mm}$ film analyzer, Model \#926, Lafayette Instrument Co., Lafayette, Indiana, U.S.A.

$\$$ Hewlett-Packard 14057A electrodes, Hewlett-Packard Medical Products Group, 175 Wyman St., Waltham, MA 02154, U.S.A.

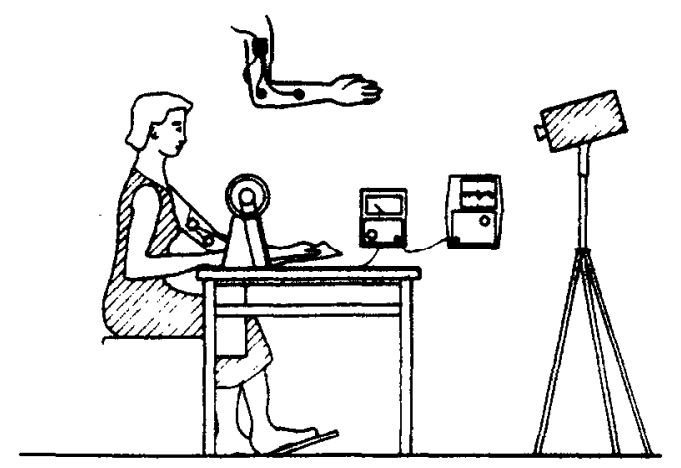

Fig. 1. Cinematography and electromyography were used to record work methods of subjects.

surface EMG of the medial side of the forearm. Active and reference monopolar electrodes+ were fixed to the skin over the midsection of the medial side of the

High CTS incidence job
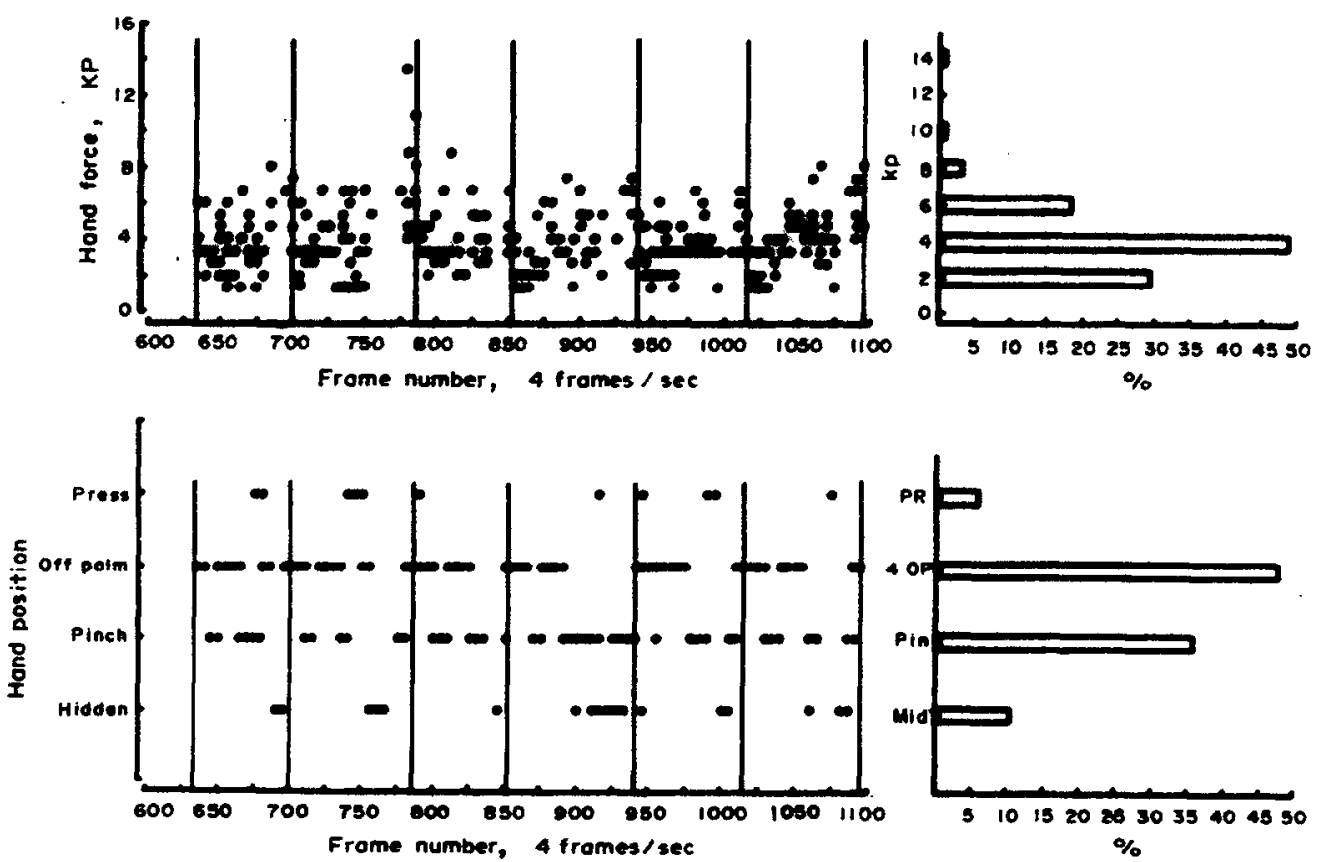

Fig. 2. Time plots and summary histograms for one operator. The vertical lines denote the beginning of new work cycles. 


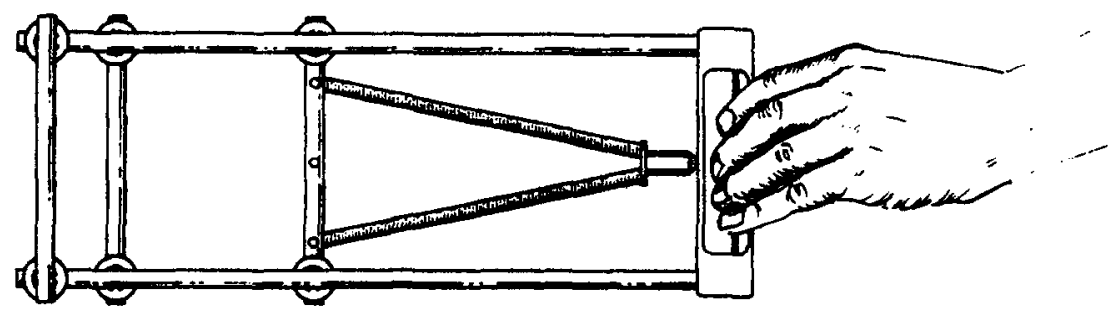

Fig. 3. Fixture used to produce desired hand forces for calibration of EMG.

forearm and over the medial epicondyle; a ground electrode was attached to the lateral epicondyle. The electrodes were plugged into a preamplifier with a gain of 30 ; the preamplifier was plugged into an a.c. voltmeter* that produced a gain between 1 and 1000 .

From work by Lippold (1952), DeVries (1968) and Bouisset (1973), it is known that the integrated EMG is empirically related to the force of exertion for a given posture. A fixture that was devised to facilitate determination of the relationship between hand force and the integrated surface EMG for various hand positions is shown in Fig. 3. The calibration device consists of two handles that are held apart by springs, which can be stretched to produce desired force levels. The subjects pinched the handles together with the desired hand position until they nearly touched while the corresponding integrated EMG level was recorded. A calibration plot then was made for each

\footnotetext{
- Heath Kit, AC Voltmeter, Model \#SM-5238, Heath Company, Benton Harbor, Michigan, U.S.A.
}

hand position of each subject as is shown in Fig. 4. For most tests across submaximal force ranges, a straight line passing through the origin fitted by method of least squares was acceptable for first order approximations of hand force. Extrapolation beyond the range of the observed calibration data is not recommended. Each subject should be calibrated before and after testing or if electrodes are replaced for any reason. To facilitate data reduction a meter displaying the integrated EMG was placed in the field of view of the camera so that both hand position and EMG were recorded in each film frame. The observed hand position then was used to select the appropriate calibration curve, which in turn was used with the EMG to estimate hand force. Sample hand forces are shown plotted in Fig. 2 with corresponding hand positions. So far, nearly one hundred subjects have been studied with this equipment.

While, so far, only hands have been studied, this methodology could be used for other parts of the body. Such a technique might be useful for industrial engineers, occupational therapists and physical educators.

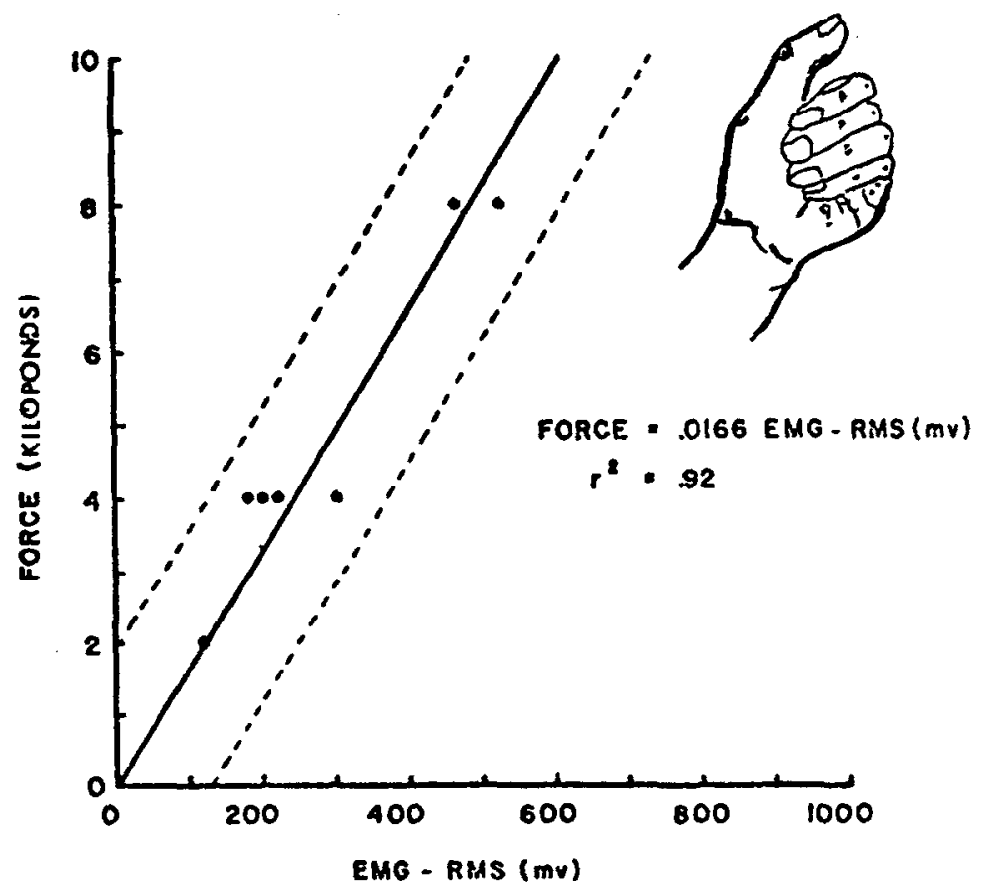

Fig. 4. A plot of hand force versus the surface EMG of the medial forearm when the fingers are flexed against the palm. 
Acknowledgements - This research was sponsored by the National Institute for Occupational Safety and Health (Grant No. 0400679) and by a private industry.

\section{REFERENCES}

Bouisset, S. (1973) EMG and Muscle force in normal motor activities. In New Developments. In Electromyography and
Clinical Neurophysiology and Clinical Neurophysioloy! (Edited by Desmedt, J. E.), pp. 547-583. Karger, Basil.

DeVries, H. (1968) Efficiency of electrical activity as a physiological measure of the functional state of muscle tissue. Am. J. phys. Med., 47, 10-22.

Lippold, $O$. (1952) The relation between integrated action potentiais in a human muscle and its isometric tension. $J$. Physiol. 117, 492-499. 